\title{
Enhancement of mobility by periodically modulating the slanting slope of a washboard potential
}

\author{
Mangal C. Mahato ${ }^{\dagger}$ and A.M. Jayannavart \\ ${ }^{\dagger}$ Department of Physics, Guru Ghasidas University, Bilaspur 495 009, Chattisgarh, India. \\ ${ }^{\star}$ Institute of Physics, Sachivalaya Marg, Bhubaneswar-751005, India
}

\begin{abstract}
Average mobility of very feebly damped particles in tilted periodic potentials is considered. Under the combined action of thermal fluctuations and small temporal modulation of the tilt of the potential the particles, in the small tilt range, become more mobile than without modulation. The enhancement of mobility depends (nonmonotonically) on the frequency of modulation. For small modulations the enhancement shows a peak as a function of frequency. This has an obvious implication on the measured voltage across a Josephson junction driven by a small amplitude alternating current of suitable frequency.
\end{abstract}

PACS numbers: 05.40.-a, 05.40.Jc, 05.60.Cd

The inertia of a particle plays spectacular role when the motion is very feebly damped even when the effective applied force is very small. The temperature of the system helps overcome the effect of damping on the motion significantly. The present work concerns motion of such particles in a periodic potential system of period $2 \pi / k$ and amplitude $V_{0}$. When a tilt as small as $F_{0}=0.07 V_{0} k$ is given to the potential at a temperature $T=0.4 V_{0} / k_{B}$, where $k_{B}$ is the Boltzmann constant, the particles make several, at times hundreds, of crossings across the potential peaks before making a "halt" in a potential well. The particles start and continue the journey (on the average downhill) again assisted by thermal fluctuations. The intervals of these continued motions and halts (that is, the intervals of the particles being in running and locked states, respectively) are not regular but must follow broad distributions. A more or less similar picture appears when the potential tilt is modulated periodically in time with amplitude $\Delta F=0.01 V_{0} k, 0.02 V_{0} k$,..etc (Fig. 1). It has been shown earlier that upon modulation the mobility of the particle exhibits hysteretic behavior. The condition of criticality of such hysteresis loops and corresponding jump distributions, which show transition between exponential to power law behavior, have been discussed in reference [1]. It is also stated there that no appreciable hysteresis loop could be obtained if the average tilt $F_{0}$ is chosen relatively far from a threshold value dependent on the product of the friction coefficient $\gamma_{0}$ and $V_{0}$. We find that if the friction coefficient becomes space dependent, and in particular periodic with the same periodicity $2 \pi / k$ but with a phase shift $\phi$ and amplitude $\lambda$, appreciable hysteresis loops can be obtained for values of $F_{0}$ roughly in the range between $0.05 V_{0} k$ and $0.14 V_{0} k$. The periodically varying friction coefficient corresponds analogously to the term representing interference between the quasiparticle tunneling and the Cooper pair tunneling across Josephson junctions [2]. The inclusion of nonuniform friction coefficient does not give qualitatively different result. However, quantitatively it makes difference. We, therefore, consider space dependent friction coefficient in our study.

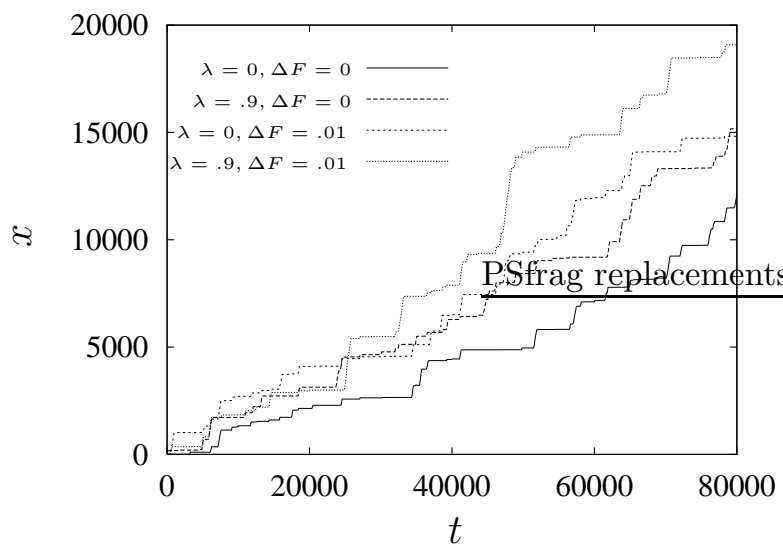

FIG. 1. Distance $x(t)$ travelled in time $(t)$. The periodicity $T_{\Omega}$ of modulation is 8000 . Note that the periodicity of the potential is only $2 \pi$. (All quantities are in dimensionless units; for example $k x$ is plotted as $x$.) Even the small vertical steps correspond to the particle travelling for hundreds of periods of the potential.

Consider the equation of motion [3],

$m \frac{d^{2} x}{d t^{2}}+\gamma(x) \frac{d x}{d t}-V_{0} k \cos (k x)-F(t)-\sqrt{ }\left(k_{B} T \gamma(x)\right) \hat{f}(t)=0$,

where $\gamma(x)=\gamma_{0}(1-\lambda \sin (k x+\phi))$ is the space dependent friction coefficient and $V(x)=-V_{0} \sin (k x)$ is the potential profile. The external force has two parts one giving the average tilt to the potential and other giving the time periodic modulation: $F(t)=F_{0}+\Delta F \cos (\Omega t)$. The fluctuating term $\hat{f}(t)$ satisfies $\langle\hat{f}(t)\rangle=0$ and $\left\langle\hat{f}(t) \hat{f}\left(t^{\prime}\right)\right\rangle=2 \delta\left(t-t^{\prime}\right)$ and may represent thermal noise at temperature $T$. Equation (1) is exactly the same as 
the Josephson junction equation except that one needs to replace $\phi$ by $\pi / 2$ [2] 4 . In our calculations we take $\lambda=0.9$ (incidentally, the choice happens to be in conformity with the experimental value [5]) and for comparison $\lambda=0$ (corresponding to uniform friction).

We solve the Langevin equation (1) (in fact, in its dimensionless form) numerically [6] to calculate the average mobility $\mu\left(=\lim _{t \rightarrow \infty}\left(\frac{x(t)}{t}\right) / F_{0}\right)$ as a function of $F_{0}$ for various values of $\Delta F$ and $\Omega=\frac{2 \pi}{T_{\Omega}}, T_{\Omega}$ is the periodicity of the external field $F(t)$. Here $x(t)$ is the distance travelled by the particle in time $t$ 7.

Fig. 2 shows the plot of $\gamma_{0} \mu$ versus $F_{0}$ for $\Delta F=0, \quad 0.01 V_{0} k, \quad 0.02 V_{0} k$ and $0.04 V_{0} k$ and choosing $T_{\Omega}$ in all these cases such that $\left(\frac{\Delta F}{V_{0} k}\right) /\left(\frac{T_{\Omega}}{m^{\cdot 5} V_{0}^{-.5} k^{-1}}\right)=$ constant $=0.00001$ (i.e., with a constant rate of change of the external field). The interesting aspect to note in the figure, which is also the main result of this paper, is that for a range of values of small $F_{0}$ (the range depending on the value of $\Delta F$ ) the average mobility is larger for $\Delta F \neq 0$ than for $\Delta F=0$. The result indicates that the mobility has been enhanced by modulating the slanting (small $F_{0}$, smaller than the known critical values [1]) slope of the periodic potential. In other words, the effective friction could be reduced below the mean friction just by modulating the potential periodically. It is worth mentioning that in tribology such problems are often sought to address and sometimes similar solutions hinted at [8]. It is also to be noted from the figure that the enhancement of mobility is larger for larger $\frac{\Delta F}{F_{0}}$. However, we restrict our attention to $\frac{\Delta F}{V_{0} k}=$ 0.01 and 0.02 which are relatively quite small compared to $\frac{F_{0}}{V_{0} k}$ of our interest where we expect mobility enhancement.

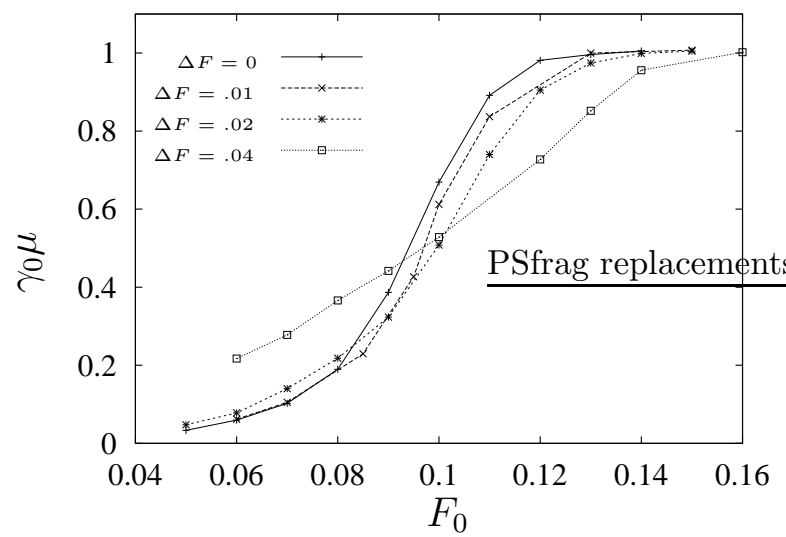

FIG. 2. $\gamma_{0} \mu$ versus $F_{0}$ for various values of $\Delta F$. The periodicity $T_{\Omega}$ for $\Delta F=.01$ is taken to be 1000 , for $\Delta F=.02$ it is 2000 , and for $\Delta F=.04$ it is 4000 so that the rate of field sweep is the same for all the three cases. (All quantities in dimensionless units)

The occurrence of the mobility enhancement can mainly be attributed to the nonlinear variation of $\gamma_{0} \mu$ as a function of $F_{0}$ with $\Delta F=0$. As can be seen from the figure the enhancement occurs where the curvature of the $\gamma_{0} \mu-F_{0}$ plot is concave upward. And this happens at lower values of $F_{0}$. At low values of $F_{0}$, during the positive half cycle of the modulating field $F(t)$ the system samples more higher velocities than it samples lower values during the negative half cycle resulting in a positive gain in the average mobility. The situation is just the reverse for higher values of $F_{0}$. The explanation is supported by the shapes of the hysteresis loops (Fig. 3) obtained because of modulation. As can be seen from the figure, for example, the hysteresis loop for $F_{0}=0.8 V_{0} k$ is more oval upward right whereas for $F_{0}=0.11 V_{0} k$ the loop is more oval downward left. However, the shapes of the loops themselves are determined by the distribution of intervals of locked and running states of the particles. And the distributions are sensitive to the values of $F_{0}$ and other parameters such as the amplitude and frequency of modulation, etc. The choice of frequency of modulation is very important for mobility enhancement.

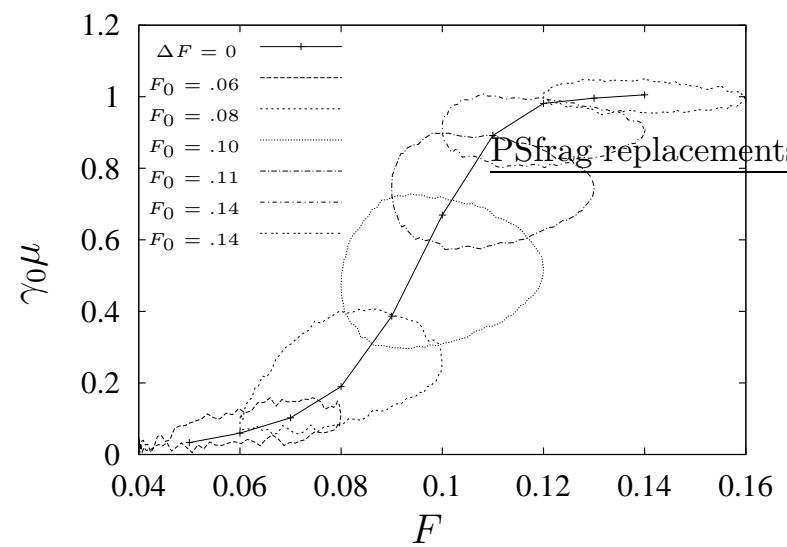

FIG. 3. $\gamma_{0} \mu$ is plotted as a function of $F_{0}$ (solid curve). The hysteresis curves are for various constant values of $F_{0}$ and $\Delta F=.02$ with period of modulation $T_{\Omega}=2000$. (All quantities in dimensionless units.)

Figure 4 shows the variation of average mobility as a function of frequency $\Omega\left(=1 / T_{\Omega}\right)$ of modulation. For large frequencies the mobility is small and close to the value for the one without modulation (shown by the horizontal line in the figure). As the frequency is decreased the mobility increases, attains a maximum and thereafter tends to decrease. The maximum enhancement for $\Delta F=0.01 V_{0} k$ is about 10 per cent whereas for $\Delta F=0.02 V_{0} k$ it is as large as about 70 per cent of the mobility obtained without modulation [9]. For $\Delta F=0.01 V_{0} k$ the curve has a distinct peak. However, for $\Delta F=0.02 V_{0} k$ the peak is not as clear. For small modulation, as the frequency goes toward zero $(\Delta F \neq 0, \Omega \rightarrow 0)$ the mobility decreases and tends to become closer to the one corresponding to without modulation $(\Delta F=0)$. But for relatively large modulation 
(e.g., $\Delta F=0.02 V_{0} k$, for $F_{0}=0.07 V_{0} k$ ) the same does not seem to be true [10]. Therefore, for relatively small amplitude modulation it is possible to maximize the enhancement of mobility by properly tuning the frequency of modulation. In the Josephson junction parlance it is reasonable to state that the sensitivity of ac SQUIDs can be improved by properly choosing the amplitude and frequency of applied alternating current [11.
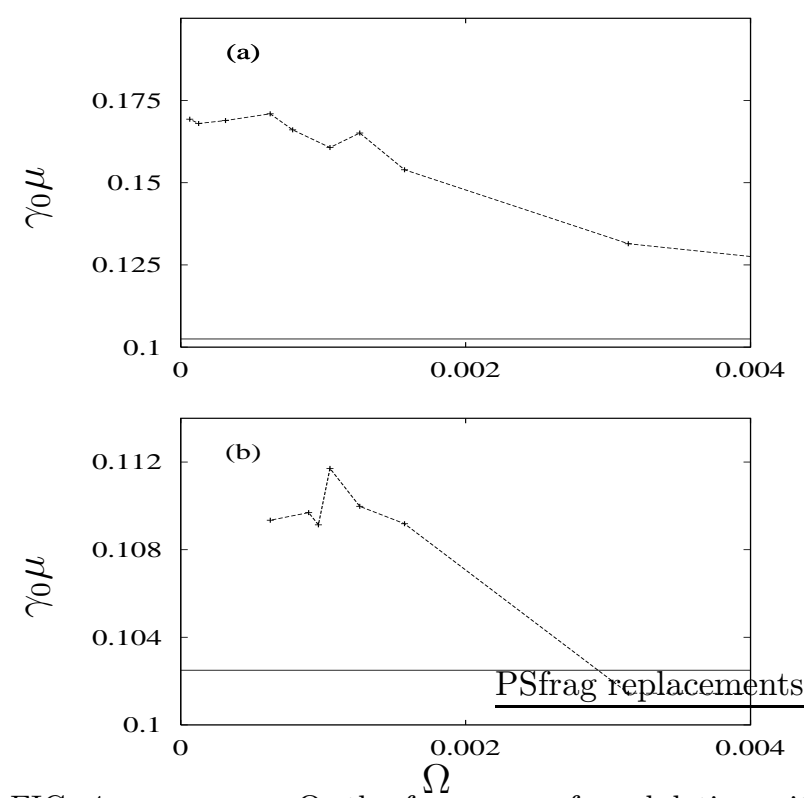

FIG. 4. $\gamma_{0} \mu$ versus $\Omega$, the frequency of modulation with $F_{0}=.07$ and (a) $\Delta F=.02$ and (b) $\Delta F=.01$. The horizontal line is drawn for reference corresponding to $\gamma_{0} \mu$ for $\Delta F=0$. (All quantities in dimensionless units.)

In the entire process of particle motion temperature plays a very important role. At zero temperature, for example, once the particle gets trapped at any one of the troughs of the potential, given the values of $F_{0}$ and $V_{0}$ considered, it will remain locked for ever making the particle effectively immobile. It is the thermal fluctuations that release the particle from the locked states into the running states [12]. As one can see from Fig. 1b of reference [1] that the mobility versus $F_{0}$ curve (for small $F_{0}$ ) changes very rapidly with temperature. Their curvatures at small $F_{0}$ too differ. One would thus expect that mobility enhancement for given values of $F_{0}, \Delta F$ and $T_{\Omega}$ will show a peaking behavior showing the phenomenon of stochastic resonance as a result of synchronization due to the interplay between external drive and thermally assisted running to locked transitions [13]. This aspect is presently being investigated.

\section{ACKNOWLEDGEMENT}

MCM thanks the Institute of Physics, Bhubaneswar for partial support and hospitality. The authors thank BRNS, DAE, India for financial support.

* email: jayan@iopb.res.in

[1] B. Borromeo, G. Constantini, and F. Marchesoni, Phys. Rev. Lett. 82, 2828 (1999).

[2] C.M. Falco, Am. J. Phys. 44, 733 (1976).

[3] A.M. Jayannavar and M.C. Mahato, Pramana J. Phys. 45, 369 (1995); cond-mat/9509059. M.C.Mahato, T.P. Pareek and A.M. Jayannavar, Int. J. Mod. Phys. B10, 3857 (1996); cond-mat/9603103.

[4] V. Ambegaokar and B.I. Halperin, Phys. Rev. Lett. 22, 1364 (1969).

[5] See the last para of Appendix B of reference [2].

[6] The equation was solved using the Runge-Kutta method of order 4: Subroutine RK4 of W.H. Press, S.A. Teukolsky, W.T. Vetterling, and B.P. Flannery, Numerical Recipes (in Fortran):The Art of Scientific Computing, Cambridge Univ. Press, 1992.

[7] To be exact we should have taken a large number of realizations of $x(t)$ to get the average mobility. However, since $t$ is very large one realization of the trajectory is assumed to be quite reasonable and adequate for calculating the average.

[8] S. Granick, Physics Today 52, (July, 1999).

[9] Mobilities on an overdamped potential system driven by additive and multiplicative dichotomous correlated noises have been investigated earlier. The constant tilt $F_{0}$ required there were large, as they should be. There too a similar situation was encountered when mobility remained large even when the relevant rate of dichotomous fluctuations was reduced to a small value. See Fig. 11 of V. Berdechevsky and M. Gitterman, Phys. Rev. E56, 6340 (1997).

[10] For a reasonably good average of mobility one needs to calculate $x(t)$ for about 5000 cycles of modulations. For scaled $T_{\Omega}=6000$ the corresponding scaled times are typically of the order of $10^{7}$ to $10^{8}$. The CPU time needed by a PII machine is more than 15 days. For larger values of $T_{\Omega}$ the averaging costs enormous computer time and is neither practical nor feasible, with facilities at our disposal, to get results for smaller frequencies. And for $\Delta F=0.02 V_{0} k$ to see the peak, if at all it has one, one either have to employ a better numerical method for the solution or a faster machine.

[11] If one chooses (see, for example, Josephson Effect Achievements and Trends, ed. by A. Barone, World Scientific, 1986) a Josephson junction of capacitance $0.5 p F$, and critical current $10^{-9}$ Coul.s $^{-1}$, the choice of $F_{0}=0.07 V_{0} k$ corresponds to dc component of current $=7 \times 10^{-11}$ Coul.s ${ }^{-1}$ and that of $\Delta F=0.01 V_{0} k$ to the modulating current amplitude of $1 \times 10^{-11}$ Coul.s $^{-1}$. The choice of $\gamma_{0}=0.035 m^{.5} V_{0}^{.5} k$ would correspond to the resistance of the Josephson junction of about 23 kilo Ohm and temperature $T=0.4 V_{0} / k_{B}$ to about $0.1 \mathrm{~K}$. It 
turns out that the voltage measured across the junction will peak at the modulating current frequency of about $0.4 \times 10^{6}$ cycles s $^{-1}$.

[12] H. Risken, The Fokker-Planck Equation (Springer, Berlin 1984), Ch. 11.
[13] L. Gammaitoni, P. Hanggi, P.Jung and F. Marchesoni, Rev. Mod. Phys. 70, 223 (1998); M.C. Mahato, and A.M. Jayannavar, Phys. Rev. E55, 6266 (1997); Physica A248, 138 (1998); M. Borromeo and F. Marchesoni, cond-mat/0009177. 